\title{
Study and Application of State Estimator for Time-Delay Systems with State Saturation Nonlinearities
}

\author{
Yeong-Jeu Sun \\ Department of Electrical Engineering, I-Shou University, Kaohsiung 840, Taiwan \\ Correspondence should be addressed to Yeong-Jeu Sun; yjsun@isu.edu.tw
}

Received 26 March 2015; Accepted 21 June 2015

Academic Editor: Emilio Insfran

Copyright (c) 2015 Yeong-Jeu Sun. This is an open access article distributed under the Creative Commons Attribution License, which permits unrestricted use, distribution, and reproduction in any medium, provided the original work is properly cited.

\begin{abstract}
The design and application of state estimator for a class of time-delay systems with state saturation nonlinearities are firstly explored. Based on the Lyapunov-Razumikhin functions, delay-dependent sufficient conditions are established to guarantee the asymptotic stability of the error between the state estimate and the true state. Besides, an upper bound of arbitrary time-varying delays is derived to assure that the resulting error can converge asymptotically to zero. Finally, applications to the secure communication and simulation results are given to demonstrate the feasibility and effectiveness of the main results.
\end{abstract}

\section{Introduction}

It is well known that time delays are frequently encountered in various physical dynamic systems and may lead to instability or oscillation. Recently, time-delay systems have been extensively studied; see, for instance, [1-8], and they are often encountered in various areas, such as chemical engineering systems, electrical system, mechanical system, biological system, transportation system, the nuclear reactor, AIDS epidemic, and systems with lossless transmission lines. However, time-delay systems are, in general, infinite dimensional systems, which make the analysis and synthesis complicated. As is well known, there have been two key approaches to deal with the problems of time-delay systems, namely, Lyapunov-Razumikhin function approach $[1,3,6]$ and Lyapunov-Krasovskii functional approach $[4,5,8]$. In general, the Lyapunov-Krasovskii approach controller design and stability analysis are more complex than the LyapunovRazumikhin approach. Besides, the Lyapunov-Krasovskii approach frequently requires the time-varying delay $h_{i}(t)$ to meet some conservational conditions such that $h_{i}(t)$ is bounded and $\dot{h}_{i}(t) \leq 1$ but the Lyapunov-Razumikhin approach only requires the time-varying delay to be bounded. To the author's knowledge, there have not been any reported results on the use of the Lyapunov-Razumikhin method for state estimator of time-delay systems with state saturation nonlinearities. Therefore it is important to develop a novel state estimate for a class of time-delay systems with state saturation nonlinearities and time-varying delays via the Lyapunov-Razumikhin technique.

On the other hand, saturation nonlinearities frequently appear in most physical systems and have drawn the attention of several researchers; reader is referred to $[2,7,9,10]$ and the references therein; for example, the states are constrained to stay within a bounded set due to physical limitations of the devices or by protection equipment. For nonlinear systems with state saturation nonlinearities, it is of great difficulty and challenge to design state estimator, in that such nonlinearities will generate a complex nonlinear system.

Since not all of internal states can be measured directly, some observing structure should be used to reconstruct the state-variables. Nevertheless, the state estimator design of dynamic systems with saturation nonlinearities is in general not as easy as that without saturation nonlinearities. Due to above-mentioned reasons, the estimator design of timedelay systems employing saturation nonlinearities is indeed an important and challenging task.

The main contributions of this paper are summarized as follows: (I) searching a suitable state estimator for a class of time-delay systems with state saturation nonlinearities to achieve the asymptotic stability of the error between the state estimate and the true state; (II) establishing delaydependent sufficient conditions to guarantee the existence of state estimator for a class of time-delay systems with 
state saturation nonlinearities and time-varying delays; (III) calculating an upper bound of arbitrary time-varying delays without destroying the state estimator; (IV) constructing an observer-based secure communication architecture to demonstrate the practicability and effectiveness of the main results.

\section{Problem Formulation and Main Results}

\section{Nomenclature}

$\mathfrak{R}^{m \times n}:=$ the set of all real $m$ by $n$ matrices,

$A^{T}:=$ the transpose of the matrix $A$,

$I:=$ the unit matrix,

$\|A\|:=$ the induced Euclidean norm of the matrix $A$, $\lambda_{\text {max }}(Q)$ (resp., $\left.\lambda_{\text {min }}(Q)\right):=$ the maximum (resp., minimum) eigenvalue of the symmetric matrix $Q$,

$Q>0:=$ the symmetric matrix $Q$ is positive and definite,

$$
\begin{aligned}
& \underline{p}:=\{1,2, \ldots, p\}, \\
& \bar{p}:=\{0,1,2, \ldots, p\} .
\end{aligned}
$$

Nonlinear time-delay systems with state saturation nonlinearities considered in this paper are described by the following state-space representation:

$$
\begin{aligned}
\dot{x}(t)= & B_{0} x(t)+\sum_{i=1}^{p} B_{i} x\left(t-h_{i}(t)\right) \\
& +\sum_{i=1}^{q}\left[E_{i} f_{\lambda_{1}, \lambda_{2}, \ldots, \lambda_{n}}\left(x\left(t-\tau_{i}(t)\right)\right)\right] \\
& +D u(t), \quad t \geq 0 \\
y(t)= & C_{0} x(t)+\sum_{i=1}^{p} C_{i} x\left(t-h_{i}(t)\right) \\
& +\sum_{i=1}^{q}\left[H_{i} f_{\lambda_{1}, \lambda_{2}, \ldots, \lambda_{n}}\left(x\left(t-\tau_{i}(t)\right)\right)\right], \\
x(t)= & \theta(t), \quad t \in[-H, 0],
\end{aligned}
$$

where $x \in \mathfrak{R}^{n \times 1}$ is the state vector, $y \in \mathfrak{R}^{r \times 1}$ is the output vector, $u \in \mathfrak{R}^{d \times 1}$ is the chaotic input vector, $h_{i}(t)^{\prime} s$, for all $i \in$ $p$, and $\tau_{i}(t)^{\prime} s$, for all $i \in q$, are arbitrary delay arguments with $0 \leq h_{i}(t) \leq H$ and $0^{-} \leq \tau_{i}(t) \leq H$ for some constant $H, B_{i}, C_{i}, E_{i}, H_{i}$, and $D$ are matrices of appropriate dimensions, and $\theta(t)$ is a given continuous vector-valued initial function. As we know, saturation nonlinearities may result in the instability or complexity of the designed system. Saturation overflow arithmetic is still one of the well-known nonlinear phenomena observed in the real world [9]. In this paper, the saturation nonlinearity $f_{\lambda_{1}, \lambda_{2}, \ldots, \lambda_{n}}(x): \mathfrak{R}^{n \times 1} \rightarrow$ $\mathfrak{R}^{n \times 1}$, with $\lambda_{i}>0$, for all $i \in \underline{n}$, is defined as follows $[2,7,10]$ :

$$
f_{\lambda_{1}, \lambda_{2}, \ldots, \lambda_{n}}(x):=\left[\begin{array}{c}
\operatorname{sat}_{\lambda_{1}}\left(x_{1}\right) \\
\operatorname{sat}_{\lambda_{2}}\left(x_{2}\right) \\
\vdots \\
\operatorname{sat}_{\lambda_{n}}\left(x_{n}\right)
\end{array}\right],
$$

with $x:=\left[\begin{array}{llll}x_{1} & x_{2} & \cdots & x_{n}\end{array}\right]^{T}$ and

$$
\operatorname{sat}_{\lambda}(z):= \begin{cases}\lambda, & z \geq \lambda \\ z, & -\lambda<z<\lambda \\ -\lambda, & z \leq-\lambda .\end{cases}
$$

Throughout this paper, the following assumption is made on systems (la), (1b), and (1c).

(A1) There exists a matrix $K \in \mathfrak{R}^{d \times r}$ such that

$$
\begin{aligned}
& A:=\left(\sum_{i=0}^{p} B_{i}\right)+D K\left(\sum_{i=0}^{p} C_{i}\right) \text { is a Hurwitz matrix; } \\
& \sqrt{\frac{\lambda_{\text {min }}^{\lambda_{\max }^{3}(P)}}{(P)}} \\
& >\quad\left(\sum_{i=1}^{p} h_{i}(t) \cdot\left\|B_{i}+D K C_{i}\right\|\right) \\
& \quad \cdot\left(\sum_{i=0}^{p}\left\|B_{i}+D K C_{i}\right\|+\sum_{i=1}^{q}\left\|E_{i}+D K H_{i}\right\|\right) \\
& \quad+\left(\sum_{i=1}^{q}\left\|E_{i}+D K H_{i}\right\|\right), \quad \forall t \geq 0
\end{aligned}
$$

where $P>0$ is the unique solution to the following Lyapunov equation:

$$
A^{T} P+P A=-2 I \text {. }
$$

Here we propose a full state estimator of the form

$$
\begin{aligned}
\dot{\hat{x}}(t)= & B_{0} \widehat{x}(t)+\sum_{i=1}^{p} B_{i} \widehat{x}\left(t-h_{i}(t)\right) \\
& +\sum_{i=1}^{q}\left[E_{i} f_{\lambda_{1}, \lambda_{2}, \ldots, \lambda_{n}}\left(\widehat{x}\left(t-\tau_{i}(t)\right)\right)\right] \\
& +D u(t)-D K[y(t)-\widehat{y}(t)], \quad t \geq 0, \\
\hat{y}(t)= & C_{0} \widehat{x}(t)+\sum_{i=1}^{p} C_{i} \widehat{x}\left(t-h_{i}(t)\right) \\
& +\sum_{i=1}^{q}\left[H_{i} f_{\lambda_{1}, \lambda_{2}, \ldots, \lambda_{n}}\left(\widehat{x}\left(t-\tau_{i}(t)\right)\right)\right],
\end{aligned}
$$

$t \geq 0$,

where $\widehat{x}(t)$ expresses the reconstructed state of the system and the constant matrix $K \in \mathfrak{R}^{d \times r}$ is defined in (A1). 
Remark 1. It has been shown in $[1,3-6,8]$ that LyapunovRazumikhin function approach and Lyapunov-Krasovskii functional approach are the key tools in the analysis of time-delayed dynamical systems. In [4], some nonnegative inequalities and Lyapunov-Krasovskii functional approach have been used to design the switching control for a class of time-delay systems. In [5], based on a new LyapunovKrasovskii functional, linear matrix inequality technique, and free weighting matrix approach, a control law has been presented to make the neutral time-delayed system robustly asymptotically stable. Besides, Razumikhin-type uniform stability has been developed in [1] to guarantee the stability of functional fractional-order time-delay systems. Meanwhile, a necessary and sufficient stability condition joining the Razumikhin and the Lyapunov-Krasovskii approaches has been provided in [6] for linear systems with several delays. The condition allows estimating the stability domains in the parameter space. On the basis of Lyapunov-Krasovskii functional approach, less conservative delay-dependent stability criteria have been proposed in [8] to guarantee the stability of the neutral time-delay system.

Remark 2. In practice, the saturation nonlinearities can be regarded as uncertainties with Lipschitz property. Besides, Lyapunov-Razumikhin function method is a useful and general approach for studying the stability of time-delay systems with uncertainties. Therefore, motivated by LyapunovRazumikhin function approach in [3], an asymptotic state estimator for time-delay systems with saturation nonlinearities of (1a), (1b), and (1c) will be presented. By the way, a sufficient condition for the asymptotic convergence to zero of the state observation error will be derived.

Now we present the first main result for the state reconstruction of systems (1a), (1b), and (1c).

Theorem 3. If condition (A1) is satisfied, systems (6a) and (6b) are an asymptotic state estimator for systems (1a), (1b), and (1c); that is, $\lim _{t \rightarrow \infty}\|x(t)-\widehat{x}(t)\|=0$.

Proof. Define

$$
\begin{aligned}
& e(t)=x(t)-\widehat{x}(t), \\
& A_{i}=B_{i}+D K C_{i}, \quad \forall i \in \bar{p}, \\
& k_{i}=E_{i}+D K H_{i}, \quad \forall i \in \underline{q}, \\
& \Delta f(t)=\sum_{i=1}^{q}\left(E_{i}+D K H_{i}\right)\left[f_{\lambda_{1}, \lambda_{2}, \ldots, \lambda_{n}}\left(x\left(t-\tau_{i}(t)\right)\right)\right. \\
& \left.\quad-f_{\lambda_{1}, \lambda_{2}, \ldots, \lambda_{n}}\left(\hat{x}\left(t-\tau_{i}(t)\right)\right)\right] .
\end{aligned}
$$

Then, from (1a), (1b), and (1c)-(9), it is easy to see that the error dynamic system is given by

$$
\begin{gathered}
\dot{e}(t)=\left(B_{0}+D K C_{0}\right) e(t)+\sum_{i=1}^{p}\left(B_{i}+D K C_{i}\right) e(t \\
\left.-h_{i}(t)\right)+\sum_{i=1}^{q}\left(E_{i}+D K H_{i}\right)
\end{gathered}
$$

$$
\begin{aligned}
& \cdot\left[f_{\lambda_{1}, \lambda_{2}, \ldots, \lambda_{n}}\left(x\left(t-\tau_{i}(t)\right)\right)\right. \\
& \left.-f_{\lambda_{1}, \lambda_{2}, \ldots, \lambda_{n}}\left(\hat{x}\left(t-\tau_{i}(t)\right)\right)\right]=A_{0} e(t) \\
& +\left[\sum_{i=1}^{p} A_{i} e\left(t-h_{i}(t)\right)\right]+\Delta f(t),
\end{aligned}
$$

with

$$
\begin{aligned}
& \|\Delta f(t)\|=\| \sum_{i=1}^{q}\left(E_{i}+D K H_{i}\right)\left[f_{\lambda_{1}, \lambda_{2}, \ldots, \lambda_{n}}\left(x\left(t-\tau_{i}(t)\right)\right)\right. \\
& \left.-f_{\lambda_{1}, \lambda_{2}, \ldots, \lambda_{n}}\left(\widehat{x}\left(t-\tau_{i}(t)\right)\right)\right]\left\|\leq \sum_{i=1}^{q}\right\| E_{i}+D K H_{i} \| \\
& \cdot\left\|x\left(t-\tau_{i}(t)\right)-\widehat{x}\left(t-\tau_{i}(t)\right)\right\|=\sum_{i=1}^{q}\left\|E_{i}+D K H_{i}\right\| \\
& \cdot\left\|e\left(t-\tau_{i}(t)\right)\right\|,
\end{aligned}
$$

in view of the global Lipschitz of $\operatorname{sat}_{\lambda}(z)$. From (10), we have

$$
\begin{aligned}
& \dot{e}(t)=A_{0} e(t)+\sum_{i=1}^{p} A_{i} e(t) \\
&- {\left[\sum_{i=1}^{p} A_{i}\left(\int_{t-h_{i}(t)}^{t} \dot{e}(z) d z\right)\right]+\Delta f(t) } \\
&=\left(\sum_{i=0}^{p} A_{i}\right) e(t)-\left\{\sum_{i=1}^{p} A_{i}\right. \\
&\left.\cdot \int_{t-h_{i}(t)}^{t}\left[\left(\sum_{i=0}^{p} A_{i} e\left(z-h_{i}(z)\right)\right)+\Delta f(z)\right] d z\right\} \\
&+\Delta f(t), \quad \forall t \geq 0, \\
& x(t)=\theta(t), \quad t \in[-H, 0] .
\end{aligned}
$$

Define the dynamic system

$$
\begin{aligned}
& \dot{y}(t)=A_{0} y(t)+\sum_{i=1}^{p} A_{i} y(t) \\
&- {\left[\sum_{i=1}^{p} A_{i}\left(\int_{t-h_{i}(t)}^{t} \dot{y}(z) d z\right)\right]+\Delta f(t) } \\
&=\left(\sum_{i=0}^{p} A_{i}\right) y(t)-\left\{\sum_{i=1}^{p} A_{i}\right. \\
&\left.\cdot \int_{t-h_{i}(t)}^{t}\left[\left(\sum_{i=0}^{p} A_{i} y\left(z-h_{i}(z)\right)\right)+\Delta f(z)\right] d z\right\} \\
&+\Delta f(t), \quad \forall t \geq 0, \\
& y(t)=e(t), \quad t \in[-H, 0], \\
& y(t)=e(-H), \quad t \in[-2 H,-H] .
\end{aligned}
$$


For brevity, let us define $y_{t}(s)=y(t+s)$, for all $s \in[-2 H$, $0]$, and $\left\|y_{t}\right\|_{s}:=\sup _{-2 H \leq r \leq 0}\|y(t+r)\|$. By comparing (13a) and (13b) with (12a) and (12b), it is easy to see that $y(t)=e(t)$, for all $t \geq 0$. Thus it can be deduced that

$$
\begin{aligned}
& \|-\sum_{i=1}^{p} A_{i} \\
& \cdot \int_{t-h_{i}(t)}^{t}\left[\left(\sum_{i=0}^{p} A_{i} y\left(z-h_{i}(z)\right)\right)+\Delta f(z)\right] d z \| \\
& \leq \sum_{i=1}^{p}\left\|A_{i}\right\| \cdot h_{i}(t) \cdot\left[\left(\sum_{i=0}^{p}\left\|A_{i}\right\| \cdot\left\|y_{t}\right\|_{s}\right)\right. \\
& \left.+\left(\sum_{i=1}^{q} k_{i} \cdot\left\|y_{t}\right\|_{s}\right)\right]=\left\{\sum_{i=1}^{p}\left\|A_{i}\right\| \cdot h_{i}(t)\right. \\
& \left..\left[\left(\sum_{i=0}^{p}\left\|A_{i}\right\|\right)+\left(\sum_{i=1}^{q} k_{i}\right)\right]\right\} \cdot\left\|y_{t}\right\|_{s}, \quad \forall t \geq 0 .
\end{aligned}
$$

Let

$$
V(y(t))=y^{T}(t) P y(t) .
$$

The time derivative of $V(y(t))$ along the trajectories of systems (13a) and (13b) is given by

$$
\begin{gathered}
\dot{V}(y(t))=y^{T}(t)\left[A^{T} P+P A\right] y(t)-2 y^{T}(t) P \sum_{i=1}^{p} A_{i} \\
\cdot \int_{t-h_{i}(t)}^{t}\left[\left(\sum_{i=0}^{p} A_{i} y\left(z-h_{i}(z)\right)\right)+\Delta f(z)\right] d z \\
+2 y^{T}(t) P \Delta f(t)=-2\|y(t)\|^{2}-2 y^{T}(t) P \sum_{i=1}^{p} A_{i} \\
\cdot \int_{t-h_{i}(t)}^{t}\left[\left(\sum_{i=0}^{p} A_{i} y\left(z-h_{i}(z)\right)\right)+\Delta f(z)\right] d z \\
+2 y^{T}(t) P \Delta f(t), \quad \forall t \geq 0 .
\end{gathered}
$$

Applying (14) to (16) yields

$$
\begin{gathered}
\dot{V}(y(t)) \leq-2\|y(t)\|^{2}+2\|y(t)\| \cdot \lambda_{\max }(P) \\
\cdot\left\{\sum_{i=1}^{p}\left\|A_{i}\right\| \cdot h_{i}(t) \cdot\left[\left(\sum_{i=0}^{p}\left\|A_{i}\right\|\right)+\left(\sum_{i=1}^{q} k_{i}\right)\right]\right\} \\
\cdot\left\|y_{t}\right\|_{s}+2\|y(t)\| \lambda_{\max }(P) \cdot\left(\sum_{i=1}^{q} k_{i}\right) \cdot\left\|y_{t}\right\|_{s} \\
=-2\|y(t)\|^{2}+2\|y(t)\| \cdot \lambda_{\max }(P) \cdot\left\{\sum_{i=1}^{p}\left\|A_{i}\right\|\right. \\
\left.\cdot h_{i}(t) \cdot\left[\left(\sum_{i=0}^{p}\left\|A_{i}\right\|\right)+\left(\sum_{i=1}^{q} k_{i}\right)\right]+\left(\sum_{i=1}^{q} k_{i}\right)\right\} \\
\cdot\left\|y_{t}\right\|_{s}, \quad \forall t \geq 0 .
\end{gathered}
$$

By (A1), (7), and (8), there exists a sufficiently small constant $\varepsilon_{1}>0$ such that

$$
\begin{array}{r}
\varepsilon_{2}:=1-\left(1+\varepsilon_{1}\right) \sqrt{\frac{\lambda_{\max }^{3}(P)}{\lambda_{\min }(P)}} \cdot\left\{\left(\sum_{i=1}^{p} h_{i}(t) \cdot\left\|A_{i}\right\|\right)\right. \\
\left.\cdot\left(\sum_{i=0}^{p}\left\|A_{i}\right\|+\sum_{i=1}^{q} k_{i}\right)+\left(\sum_{i=1}^{q} k_{i}\right)\right\}>0, \quad \forall t \geq 0 .
\end{array}
$$

In the spirit of Theorem 4.2 in [3], with $p(s)=\left(1+\varepsilon_{1}\right)^{2} s$, we suppose that

$$
\begin{aligned}
& y^{T}(t+r) P y(t+r)<\left(1+\varepsilon_{1}\right)^{2} y^{T}(t) P y(t), \\
& \forall-2 H \leq r \leq 0 ;
\end{aligned}
$$

this implies that

$$
\begin{array}{r}
\lambda_{\text {min }}(P)\|y(t+r)\|^{2}<\left(1+\varepsilon_{1}\right)^{2} \lambda_{\text {max }}(P)\|y(t)\|^{2}, \\
\forall-2 H \leq r \leq 0 .
\end{array}
$$

This shows that

$$
\begin{aligned}
\|y(t+r)\|<\left(1+\varepsilon_{1}\right) \sqrt{\frac{\lambda_{\max }(P)}{\lambda_{\min }(P)}}\|y(t)\|, & \\
& \forall-2 H \leq r \leq 0 .
\end{aligned}
$$

Substituting (21) into (17), it can be shown that

$$
\begin{aligned}
& \dot{V}(y(t)) \leq-2\|y(t)\|^{2}+2\|y(t)\| \cdot \lambda_{\max }(P) \\
& \cdot\left\{\left(\sum_{i=1}^{p}\left\|A_{i}\right\| \cdot h_{i}(t)\right)\left[\left(\sum_{i=0}^{p}\left\|A_{i}\right\|\right)+\left(\sum_{i=1}^{q} k_{i}\right)\right]\right. \\
& \left.+\left(\sum_{i=1}^{q} k_{i}\right)\right\} \cdot\left(1+\varepsilon_{1}\right) \cdot \sqrt{\frac{\lambda_{\max }(P)}{\lambda_{\max }(P)}} \cdot\|y(t)\| \\
& =-2 \varepsilon_{2}\|y(t)\|^{2}, \quad \forall t \geq 0,
\end{aligned}
$$

in view of (18). Thus, by Theorem 4.2 in [3] with (15) and (22), we conclude that systems (13a) and (13b) and systems (12a) and (12b) are both globally asymptotically stable with

$$
\lim _{t \rightarrow \infty}\|e(t)\|=\lim _{t \rightarrow \infty}\|x(t)-\widehat{x}(t)\|=0
$$

This completes our proof.

Remark 4. By Theorem 3, an upper bound of arbitrary timevarying delays without destroying the state estimator is given by $H<\bar{H}$, where 


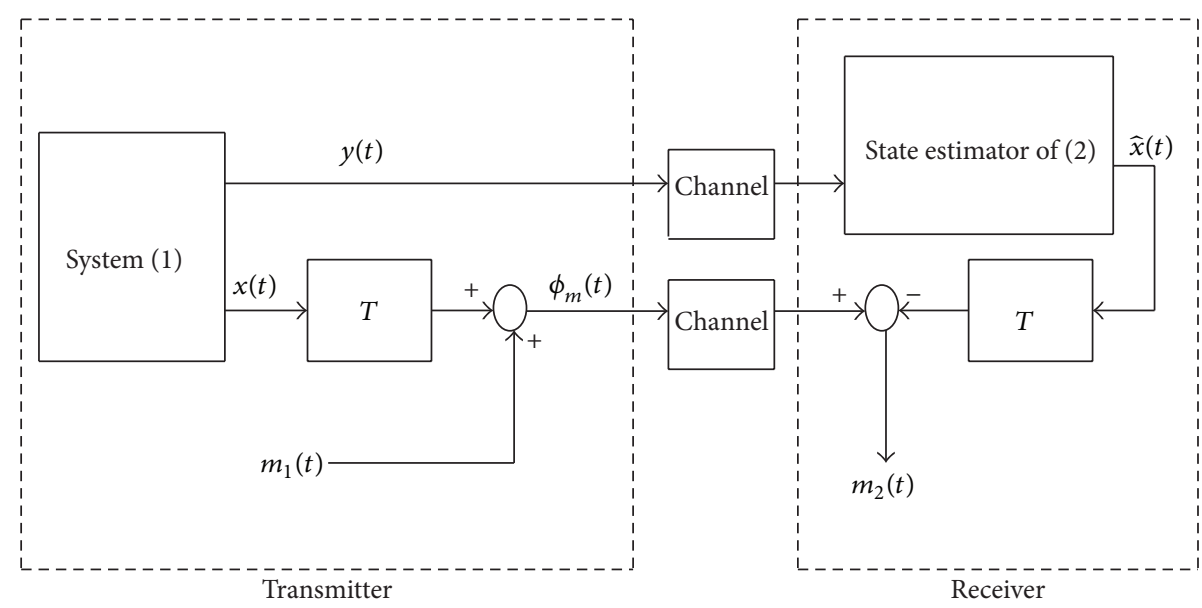

FIGURE 1: Secure communication system $\left(m_{1}(t)\right.$ is the message vector and $m_{2}(t)$ is the recovered vector).

$$
\begin{aligned}
& \bar{H}= \begin{cases}\frac{\alpha_{4}-\alpha_{3}}{\alpha_{1}\left(\alpha_{2}+\alpha_{3}\right)}, & \text { if } \alpha_{1}\left(\alpha_{2}+\alpha_{3}\right) \neq 0, \alpha_{4}>\alpha_{3}, \\
\infty, & \text { if } \alpha_{1}\left(\alpha_{2}+\alpha_{3}\right)=0, \alpha_{4}>\alpha_{3},\end{cases} \\
& \qquad \alpha_{1}=\sum_{i=1}^{p}\left\|B_{i}+D K C_{i}\right\|, \alpha_{2}=\sum_{i=0}^{p}\left\|B_{i}+D K C_{i}\right\|, \alpha_{3}=\sum_{i=1}^{q}\left\|E_{i}+D K H_{i}\right\|, \alpha_{4}=\sqrt{\frac{\lambda_{\text {min }}(P)}{\lambda_{\text {max }}^{3}(P)}} .
\end{aligned}
$$

\section{Application to Secure \\ Communication System}

Motivated by the time-delay systems (1a), (1b), and (1c) with their state estimator of $(6 a)$ and $(6 b)$, let us consider the following secure communication system and the architecture of such a system is shown in Figure 1.

\section{Transmitter:}

$$
\begin{aligned}
\dot{x}(t)= & B_{0} x(t)+\sum_{i=1}^{p} B_{i} x\left(t-h_{i}(t)\right) \\
& +\sum_{i=1}^{q}\left[E_{i} f_{\lambda_{1}, \lambda_{2}, \ldots, \lambda_{n}}\left(x\left(t-\tau_{i}(t)\right)\right)\right] \\
& +D u(t), \quad t \geq 0, \\
y(t)= & C_{0} x(t)+\sum_{i=1}^{p} C_{i} x\left(t-h_{i}(t)\right) \\
& +\sum_{i=1}^{q}\left[H_{i} f_{\lambda_{1}, \lambda_{2}, \ldots, \lambda_{n}}\left(x\left(t-\tau_{i}(t)\right)\right)\right],
\end{aligned}
$$

$t \geq 0$,

$\phi_{m}(t)=T x(t)+m_{1}(t)$, where $m_{1}(t) \in \mathfrak{R}^{v \times 1}$ is the message vector, $T \in \mathfrak{R}^{v \times n}$, and systems (25a) and (25b) are the same as systems (1a) and (1b), respectively.

Receiver:

$$
\begin{aligned}
\dot{\hat{x}}(t)= & B_{0} \widehat{x}(t)+\sum_{i=1}^{p} B_{i} \widehat{x}\left(t-h_{i}(t)\right) \\
& +\sum_{i=1}^{q}\left[E_{i} f_{\lambda_{1}, \lambda_{2}, \ldots, \lambda_{n}}\left(\widehat{x}\left(t-\tau_{i}(t)\right)\right)\right] \\
& +D u(t)-D K[y(t)-\widehat{y}(t)], \quad t \geq 0, \\
\hat{y}(t)= & C_{0} \widehat{x}(t)+\sum_{i=1}^{p} C_{i} \widehat{x}\left(t-h_{i}(t)\right) \\
& +\sum_{i=1}^{q}\left[H_{i} f_{\lambda_{1}, \lambda_{2}, \ldots, \lambda_{n}}\left(\widehat{x}\left(t-\tau_{i}(t)\right)\right)\right], \\
m_{2}(t)= & \phi_{m}(t)-T \widehat{x}(t),
\end{aligned}
$$

where dynamics of (26a) and (26b) are the same as those of (6a) and (6b), respectively. In addition, $m_{2}(t) \in \mathfrak{R}^{v \times 1}$ is the signal recovered from $m_{1}(t)$. It is worthwhile to note that our proposed secure communication is motivated by the timedelay systems (1a), (1b), and (1c) with their state estimator 
of (6a) and (6b). For any information vector $m_{1}(t)$ in the transmitter system, the objective of secure communication system is to recover the message $m_{2}(t)$ in the receiver system.

Theorem 5. The global synchronization of signals $m_{1}(t)$ and $m_{2}(t)$ can be realized by the transmitters $(25 a),(25 b)$, and $(25 c)$ with the receiver of (26a), (26b), and (26c), provided that (A1) is satisfied.

Proof. By Theorem 3 with (23)-(26c), one can see that

$$
\begin{aligned}
& \lim _{t \rightarrow \infty}\left\|m_{1}(t)-m_{2}(t)\right\| \\
& \quad=\lim _{t \rightarrow \infty}\left\|\phi_{m}(t)-T x(t)-\phi_{m}(t)+T \widehat{x}(t)\right\| \\
& \quad \leq \lim _{t \rightarrow \infty}\|T\| \cdot\|e(t)\|=0 .
\end{aligned}
$$

This implies that one can recover the information $m_{1}(t)$ in the receiver system. This completes our proof.

\section{Illustrative Example with Numerical Simulations}

Example. Consider the transmitter system of (25a), (25b), and $(25 \mathrm{c})$ with parameters as follows:

$$
\begin{aligned}
p & =q=2, \\
r & =1, \\
H & =10 \\
m_{1}(t) & =3 \sin (0.7 t)+3 \cos (0.5 t)+e^{-0.01 t}, \\
B_{0} & =\left[\begin{array}{cc}
-0.1 & -2 \\
0.1 & 0
\end{array}\right], \\
B_{1} & =\left[\begin{array}{cc}
-2 & -2 \\
0 & 0
\end{array}\right], \\
B_{2} & =\left[\begin{array}{cc}
0 & 2 \\
-0.1 & -0.1
\end{array}\right], \\
E_{1} & =\left[\begin{array}{cc}
2 & 0 \\
0 & 0.1
\end{array}\right], \\
C_{1} & =\left[\begin{array}{ll}
1 & 1
\end{array}\right], \\
E_{2} & =\left[\begin{array}{cc}
-2 & 2 \\
0.1 & 0.1
\end{array}\right], \\
D & =\left[\begin{array}{cc}
-1 \\
0
\end{array}\right],
\end{aligned}
$$

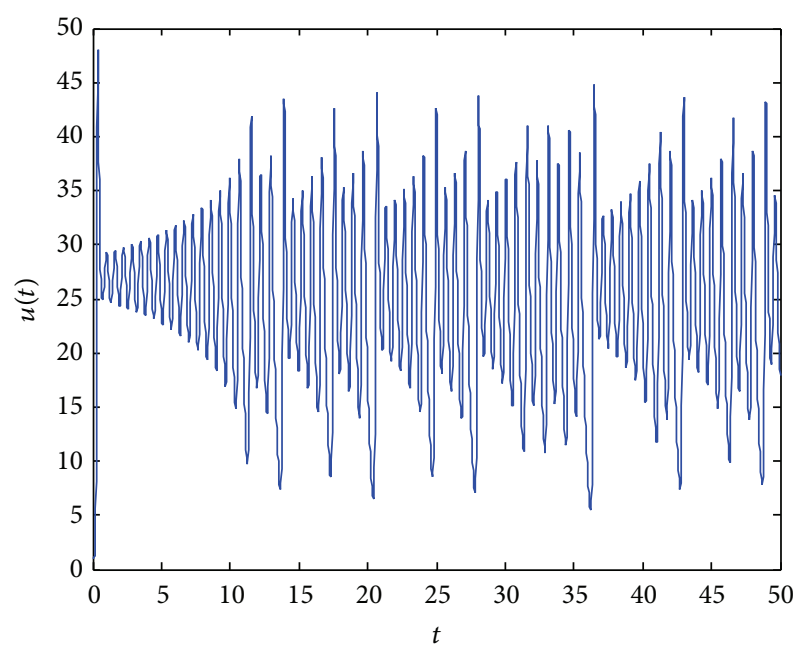

FIgURE 2: Signal of $u(t)$.

$$
\begin{aligned}
C_{2} & =\left[\begin{array}{ll}
0 & -1
\end{array}\right], \\
H_{1} & =\left[\begin{array}{ll}
1 & 0
\end{array}\right], \\
H_{2} & =\left[\begin{array}{ll}
-1 & 1
\end{array}\right], \\
T & =\left[\begin{array}{ll}
1 & -1
\end{array}\right] .
\end{aligned}
$$

Furthermore, the input $u(t)$ is a Lorenz chaotic signal, shown in Figure 2. Condition (A1) is evidently satisfied if we let $K=$ -2 . Consequently, by Theorem 3, we conclude that the state estimator of systems (25a)-(25b) is of existence and a suitable state estimator is given by (26a)-(26b). Additionally, the real information $m_{1}(t)$, the recovered information $m_{2}(t)$, and the error signal are depicted in Figures 3-5, respectively. From the foregoing simulations results, it is seen that the global synchronization of signals $m_{1}(t)$ and $m_{2}(t)$ can be achieved by the transmitters (25a), (25b), and (25c) with the receiver of (26a), (26b), and (26c). By the way, from Remark 4, an upper bound of arbitrary time-varying delays without destroying the state estimator is given by $H<\bar{H}=10.2$.

Remark 6. It should be emphasized that there exist an infinite number of secure communication systems of (25a), (25b), and (25c) with (26a), (26b), and (26c) since there exist infinitely many systems (1a), (1b), and (1c) satisfying the condition of (A1).

\section{Conclusion}

In this paper, the design and application of state estimator for a class of time-delay systems with state saturation nonlinearities have been firstly investigated. Based on the Lyapunov-Razumikhin functions, sufficient conditions have been established to guarantee the asymptotic stability of the error between the state estimate and the true state. Besides, an upper bound of arbitrary time-varying delays has been derived to guarantee that the resulting error can converge 


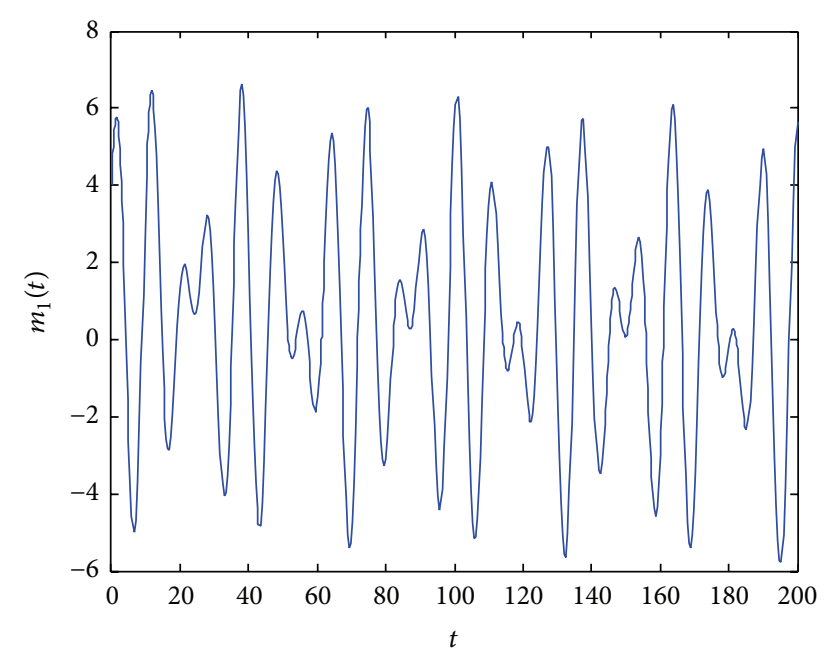

FIGURE 3: Real information of $m_{1}(t)$ described in the transmitter of (25a), (25b), and (25c).

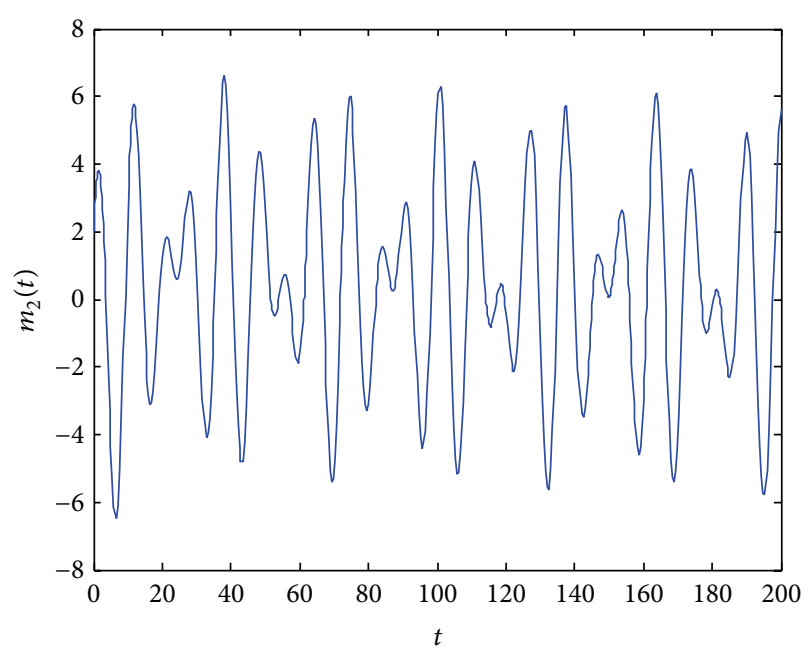

FIgURE 4: Recovered information of $m_{2}(t)$ described in the receiver of (26a), (26b), and (26c).

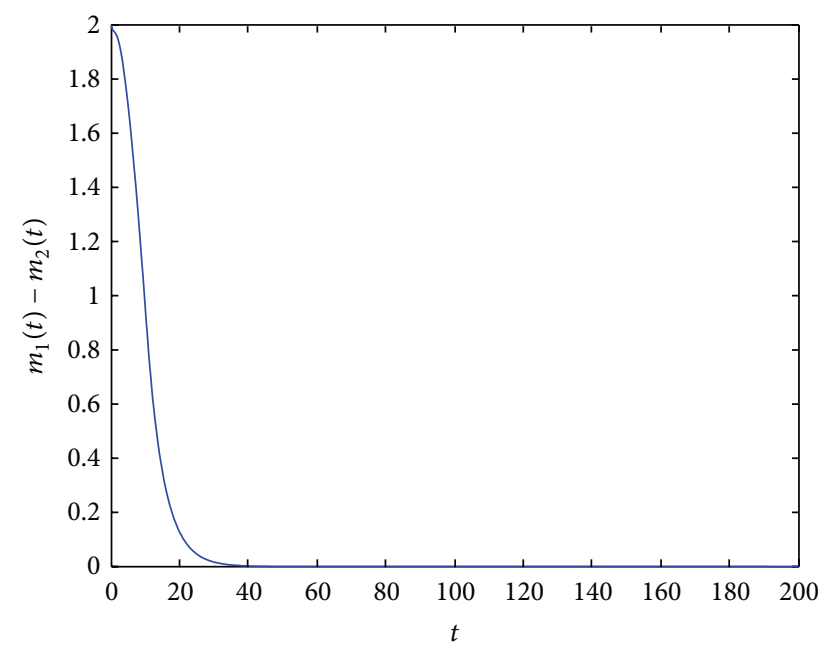

FIGURE 5: Error signal of $m_{1}(t)-m_{2}(t)$. asymptotically to zero. Applications of the proposed state estimator technique to secure communication as well as some numerical simulations have also been presented to demonstrate the practicability and effectiveness of the main results.

\section{Conflict of Interests}

The author declares that there is no conflict of interests regarding the publication of this paper.

\section{Acknowledgment}

The author thanks the Ministry of Science and Technology of China for supporting this work under Grant MOST-103-2221E-214-044.

\section{References}

[1] B. Chen and J. Chen, "Razumikhin-type stability theorems for functional fractional-order differential systems and applications," Applied Mathematics and Computation, vol. 254, no. 1, pp. 63-69, 2015.

[2] L. Fan, "Iterative learning and adaptive fault-tolerant control with application to high-speed trains under unknown speed delays and control input saturations," IET Control Theory \& Applications, vol. 8, no. 9, pp. 675-687, 2014.

[3] J. K. Hale and S. M. V. Lunel, Introduction to Functional Differential Equations, Springer, New York, NY, USA, 1993.

[4] C.-H. Lien, K.-W. Yu, L.-C. Wu, L.-Y. Chung, and J.-D. Chen, "Robust $\mathrm{H}_{\infty}$ image switching control and switching signal design for uncertain discrete switched systems with interval time-varying delay," Journal of the Franklin Institute, vol. 351, no. 1, pp. 565-578, 2014.

[5] K. Mathiyalagan, R. Sakthivel, and J. H. Park, "Robust reliable control for neutral-type nonlinear systems with time-varying delays," Reports on Mathematical Physics, vol. 74, no. 2, pp. 181203, 2014.

[6] I. V. Medvedeva and A. P. Zhabko, "Synthesis of Razumikhin and Lyapunov-Krasovskii approaches to stability analysis of time-delay systems," Automatica, vol. 51, pp. 372-377, 2015.

[7] B. Zhou, Z.-Y. Li, and Z. Lin, "Stabilization of discrete-time systems with multiple actuator delays and saturations," IEEE Transactions on Circuits and Systems. I. Regular Papers, vol. 60, no. 2, pp. 389-400, 2013.

[8] J. Sun and G. P. Liu, "On improved delay-dependent stability criteria for neutral time-delay systems," European Journal of Control, vol. 15, no. 6, pp. 613-623, 2009.

[9] A. Dey and H. Kar, "An LMI based criterion for the global asymptotic stability of 2-D discrete state-delayed systems with saturation nonlinearities," Digital Signal Processing, vol. 22, no. 4, pp. 633-639, 2012.

[10] J. H. Fan, Y. M. Zhang, and Z. Q. Zheng, "Robust fault-tolerant control against time-varying actuator faults and saturation," IET Control Theory \& Applications, vol. 6, no. 14, pp. 2198-2208, 2012. 


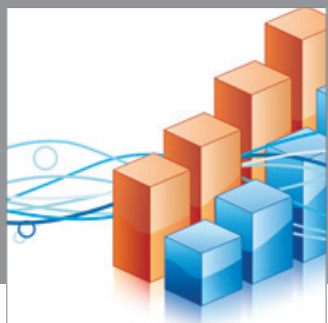

Advances in

Operations Research

mansans

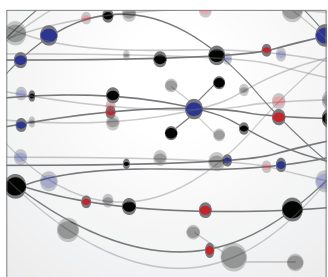

The Scientific World Journal
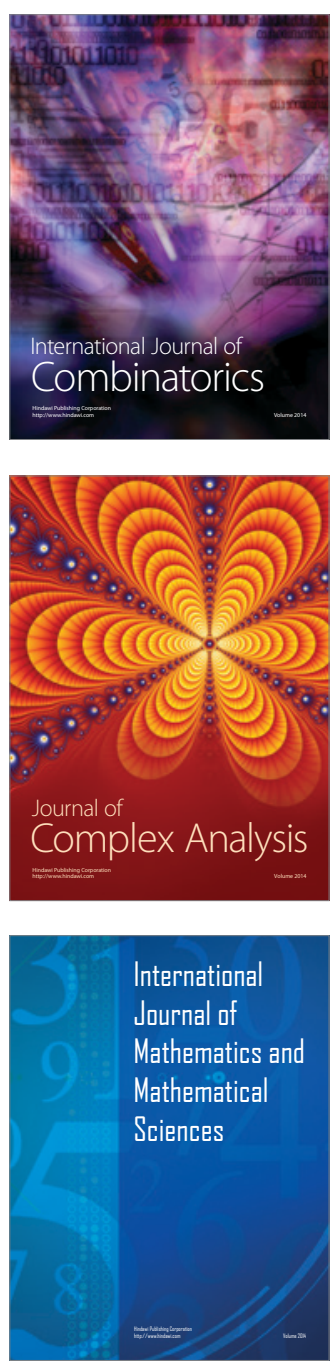
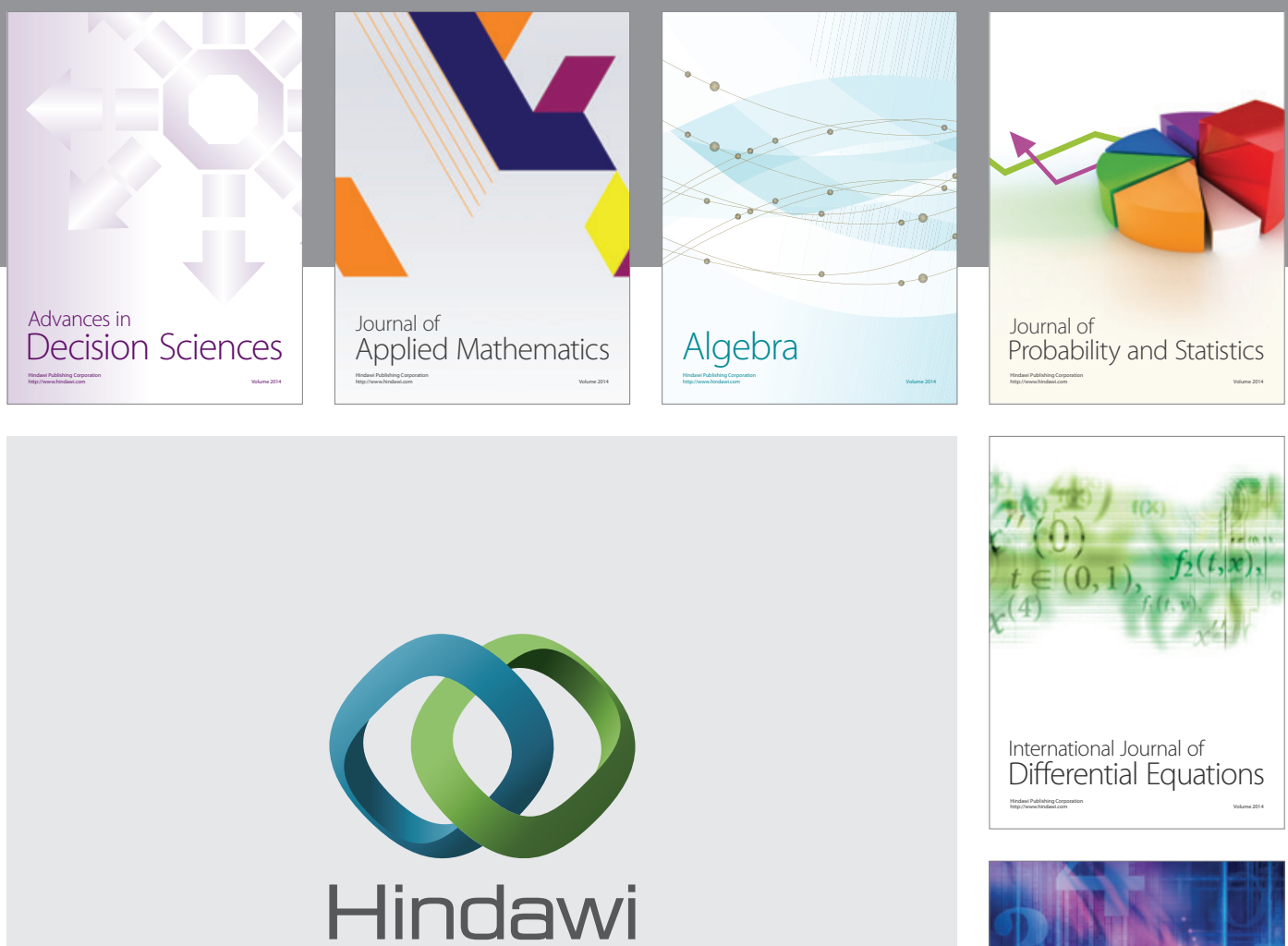

Submit your manuscripts at http://www.hindawi.com
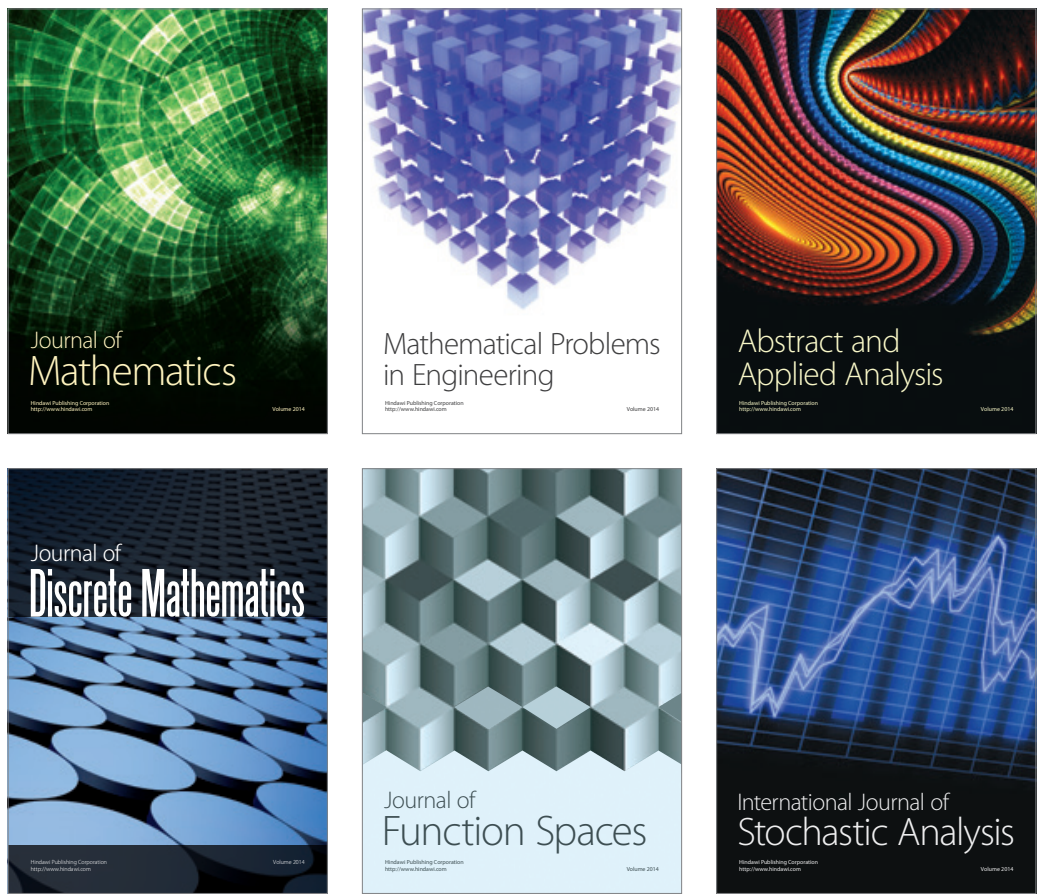

Journal of

Function Spaces

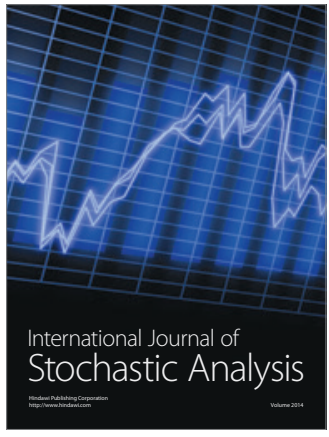

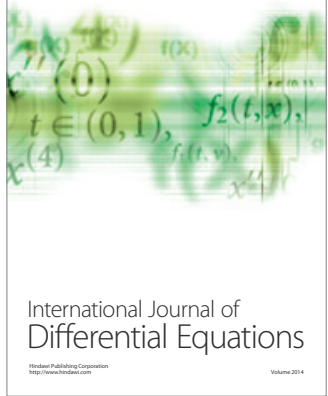
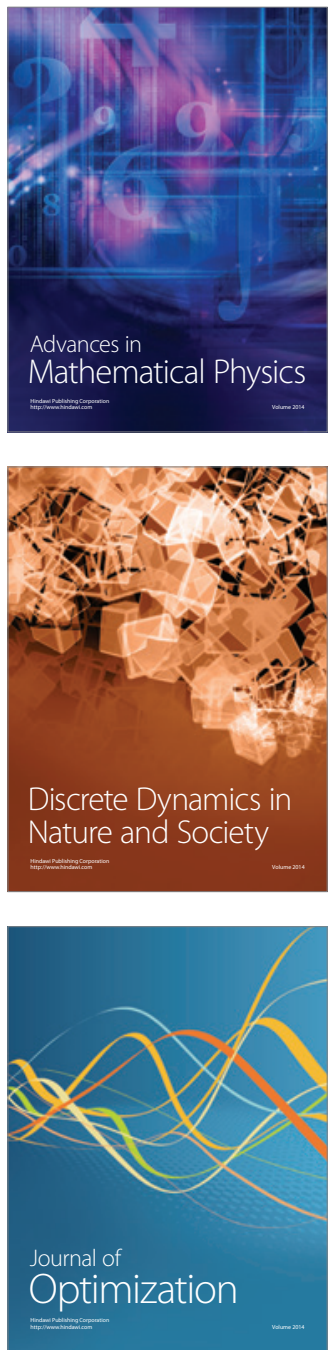